\title{
D-Test - A Useful Tool in Detection of Clindamycin Resistance among Staphylococcal Isolates in a Tertiary Care Hospital
}

\author{
Kanaga Priya Muthiah and T. Susitha* \\ ${ }^{1}$ Tirunelveli Medical College, Tirunelveli, India \\ ${ }^{2}$ Kanyakumari Government Medical College, India \\ *Corresponding author
}

\section{A B S T R A C T}

\begin{tabular}{|l|}
\hline Ke y w or d s \\
$\begin{array}{l}\text { Staphylococcus } \\
\text { aureus, CONS, } \\
\text { Inducible } \\
\text { clindamycin } \\
\text { resistance, D-test }\end{array}$ \\
\hline Article Info \\
\hline $\begin{array}{l}\text { Accepted: } \\
\text { 10 May 2019 } \\
\text { Available Online: } \\
\text { 10 June } 2019\end{array}$ \\
\hline
\end{tabular}

\section{Introduction}

Staphylococcus aureus and CoagulaseNegative Staphylococci (CONS) are recognized as important agents responsible for nosocomial and community acquired infections. In this era of super bugs, increasing antibiotic resistance is of more concern and selection of antibiotics to treat these infections has become more important.Rate of Methicillin Resistant Staphylococcus aureus (MRSA) has dramatically increased in recent years. ${ }^{1,2}$ This has led to renewed interest in the use of macrolide -lincosamide -streptogramin (MLSB) antibiotics.
To demonstrate inducible clindamycin resistance among the staphylococcal isolates from various clinical samples by D- test. A total of 125 Staphylococcus aureus and Coagulase Negative Staphylococcus (CONS) isolates were collected from clinical samples. All the samples were tested for inducible clindamycin resistance by using the D -test as per CLSI guidelines. In this study, $11 \%$ inducible clindamycin resistant staphylococcal isolates (iMLSb), and $9 \%$ of Constitutive resistance (cMLSb) and 46\% MS phenotype were detected and $34 \%$ were susceptible to both erythromycin and clindamycin (S phenotype) among the total isolates. The inducible clindamycin resistance in this study was $11 \%$. The is a very convenient test to perform. It can be done along with our routine antibiotic reporting is mandatory for a successful treatment outcome.

Clindamycin is preferred for treating these infections because of its low cost, fewer severe side effects, availability of oral and parenteral forms, lack of need for renal adjustments, good tissue penetration and ability to directly inhibit toxin production. Clindamycin' furthermore, is a useful choice in cases of penicillin allergy. ${ }^{3}$

Macrolides, Lincosamides and Streptogramin (MLS) antibiotics are structurally unrelated; however, they are related microbiologically because of their similar mode of action. MLS antibiotics inhibit bacterial protein synthesis by binding to 23s rRNA (ribosomal RNA), which is a part of the large ribosomal subunit. 
They have a spectrum of activity directed against Gram-positive cocci and intra-cellular bacteria such as chlamydiae and rickettsiae ${ }^{4}$

Resistance to MLS antibiotics occur either through target site modification, efflux of antibiotics, or drug modification. ${ }^{5}$ In targetsite modification, methylation of the A2058 residue, located in the conserved domain $\mathrm{V}$ of 23s rRNA, takes place, which leads to cross resistance and results in the formation of the phenotype of resistance pattern known as $\mathrm{MLS}_{\mathrm{B}}$. This phenotype is encoded by erythromycin ribosome methylases ( $\mathrm{rm})$ genes that have been reported from a wide variety of microorganisms. ${ }^{6}$

The expression of the MLS в phenotype can be constitutive (MLS ${ }_{\text {B }}$ c) or inducible MLS $_{B}$ i). Inducible resistance is observed when the inactive mRNA produced by the production of methylases becomes active in the presence of an inducer, while active methylase mRNA is produced in strains where constitutive expression is seen. ${ }^{5}$ The strains carrying the inducible $\mathrm{erm}$ gene are resistant to the inducer and remain susceptible to non inducer macrolides and lincosamides. Low levels of erythromycin is an inducer of the MLS ${ }_{B} i$ phenotype, which forms the basis of the Dtest $^{7}$. A second mechanism of resistance is due to active efflux pump encoded by msrA gene. They are the MS phenotype ${ }^{4}$ Strains with inducible clindamycin resistance are not detectable by the routine antimicrobial susceptibility tests. They appear to be erythromycin resistant and clindamycin susceptible in vitro. In these cases management with clindamycin will not be effective. To overcome this, the Clinical and Laboratory Standards Institute (CLSI) has recommended the erythromycin clindamycin disc approximation test ( $\mathrm{D}$ - test $)^{\text {) }} 8$ This study was done to find out the prevalence of erythromycin induced clindamycin resistance in Staphylococcus aureus and coagulase negative Staphylococcus (CONS) isolates using this valuable, simple, reliable D-test.

\section{Materials and Methods}

This cross-sectional study was conducted in a tertiary care hospital in Tirunelveli after obtaining ethical committee approval. A total of 125 isolates of staphylococcus species from various clinical samples like pus, sputum, urine, blood, and body fluids were tested. The specimens were cultured on Nutrient agar, Blood agar and MacConkey agar plates and incubated aerobically at $37^{\circ} \mathrm{C}$ for 24 hours. The isolates were identified by using conventional laboratory methodscolony morphology, gram staining, catalase, coagulase test and other standard biochemical tests. ${ }^{10}$ Antibiotic sensitivity testing was performed by Kirby - Bauer disc diffusion method on Mueller - Hinton agar plates using the following antibiotics: Ciprofloxacin $(5 \mu \mathrm{gm})$, clindamycin $(2 \mu \mathrm{gm})$, gentamicin $(10 \mu \mathrm{gm})$, erythromycin $(15 \mu \mathrm{gm})$, vancomycin

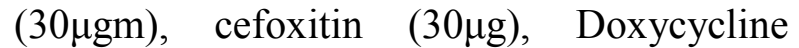
(30 $\mu \mathrm{gm}), \quad$ Co-Trimoxazole $\quad(1.25 / 23.75$ $\mu \mathrm{gm})$.Results were interpreted according to the CLSI guidelines. ${ }^{11}$ Antibiotic discs were procured from HiMedia India, Private Limited. Staphylococcus aureus ATCC 25923 was used for quality contro $1^{12}$. Cefoxitin disc was used as a surrogate marker for detecting methicillin resistance.

\section{D- Test}

The susceptibility testing was performed among the isolates by Kirby -Bauer disc diffusion method on Mueller- Hinton agar plates inoculated with 0.5 Mac Farlands standard bacterial suspension. Erythromycin $(15 \mu \mathrm{g})$ disc was placed at a distance of $15 \mathrm{~mm}$ (edge to edge separation) from Clindamycin $(2 \mu \mathrm{g})$ disc on the same plate and were incubated at $37^{0} \mathrm{C}$ overnight $^{5}$ (Figure 1). 


\section{Interpretation of D-test}

Four different phenotypes were interpreted as follows.

\section{Inducible iMLS $S_{B}$ phenotype}

Isolates showing resistance to erythromycin (zone size $\leq 13 \mathrm{~mm}$ ) and sensitive to clindamycin (zone size $\geq 21 \mathrm{~mm}$ ) with a $\mathrm{D}$ shaped zone of inhibition around clindamycin with flattening towards erythromycin disc.-Dtest positive. Same inhibitory diameter as above with no D shaped zone-D-test negative.

Four different phenotypes were interpreted as follows.

1. Inducible iMLS B $_{B}$ phenotype - Isolates showing resistance to erythromycin (zone size $\leq 13 \mathrm{~mm}$ ) and sensitive to clindamycin (zone size $\geq 21 \mathrm{~mm}$ ) with a D shaped zone of inhibition around clindamycin with flattening towards erythromycin disc.-Dtest positive. Same inhibitory diameter as above with no D shaped zone-D-test negative.

2. Constitutive $\mathbf{c M L S}_{\mathbf{B}}$ phenotype - Isolates showing resistance to both erythromycin (zone size $\leq 13 \mathrm{~mm}$ ) and clindamycin (zone size $\leq 14 \mathrm{~mm}$ ) with circular shape of zone of inhibition around clindamycin.

\section{MS phenotype - Isolates showing} resistance to erythromycin (zone size $\leq$ $13 \mathrm{~mm})$ while being sensitive to clindamycin (zone size $\geq 21 \mathrm{~mm}$ ) with a circular zone of inhibition around clindamycin.

4. S phenotype-Isolates showing sensitivity to both erythromycin zone size $\geq 23 \mathrm{~mm}$ and clindamycin $\geq 21 \mathrm{~mm} .{ }^{13}$

\section{Results and Discussion}

Among the 125 isolates studied, 74 (59\%) were Staphylococcus aureus and $51(41 \%)$ were COagulase Negative Staphylococci (CONS). Of the 74 Staphylococcus aureus strains, 51 (69\%) were Methicillin Resistant Staphylococcus aureus (MRSA) and 23 (31\%) were Methicillin Sensitive Staphylococcus aureus (MSSA). Out of 51 CONS, 46 (90\%) were Methicillin Resistant Coagulase Negative Staphylococci (MRCONS) and 5 (10\%) were Methicillin Sensitive COagulase Negative Staphylococci (MSCONS) (Table 1).

In the present study, out of the total 125 staphylococcal isolates 14 (11\%) inducible clindamycin resistant strains was detected. Staphylococcus aureus isolates were 74 among them MRSA isolates were 51 and MSSA isolates were 23. Among the 51 MRSA isolates $8(16 \%)$ were iMLSB, 6 (12\%) were cMLSb, 22 (43\%) MS phenotype and 15 (29\%) S phenotype. Among the 23 (18\%) MSSA isolates $1(5 \%)$ was iMLSB, there was no cMLSb isolate detected, 10 (44\%) MS phenotype and 12 (51\%) S phenotype.

Out of the total 51 CONS isolates 46 (37\%) were MRCONS and 5 (4\%) MSCONS. Among the 46 (37\%) MRCONS isolates 5 (11\%) were iMLSB, 5 (11\%) were cMLSb, 23 (50\%) MS phenotype and 13 (28\%)S phenotype. Among the 5 (4\%) MSCONS isolates there was no cMLSb and iMLSB, isolate detected, 2 (40\%)was MS phenotype and 3(60\%) S phenotype. In this study out of the total 125 staphylococcal were $14(11 \%)$ iMLSB, 11 (9\%) were cMLSb, 57 (46\%) MS phenotype and $43(34 \%)$ S Phenotype (Table 2). 
Table.1 Distribution of methicillin sensitive and resistant Staphylococcus aureus and cons

\begin{tabular}{|l|l|l|l|}
\hline \multicolumn{2}{|l|}{ Staphylococcus aureus $(\mathbf{n = 7 4})$} & CONS $(\mathbf{n}=\mathbf{5 1})$ \\
\hline MRSA & $51(69 \%)$ & MRCONS & $5(10 \%)$ \\
\hline MSSA & $23(31 \%)$ & MSCONS & $46(90 \%)$ \\
\hline Total $\mathbf{n = 1 2 5}$ & $74(100 \%)$ & & $51(100 \%)$ \\
\hline
\end{tabular}

Table.2 Distribution of phenotypes among methicillin sensitive and resistant Staphylococcus aureus and cons

\begin{tabular}{|l|l|l|l|l|l|}
\hline Organism & $\begin{array}{l}\text { Total } \\
\text { Isolates }\end{array}$ & iMLSb & cMLSb & $\begin{array}{l}\text { MS } \\
\text { Phenotype }\end{array}$ & $\begin{array}{l}\text { S } \\
\text { Phenotype }\end{array}$ \\
\hline MRSA & $51(41 \%)$ & $8(16 \%)$ & $6(12 \%)$ & $22(43 \%)$ & $15(29 \%)$ \\
\hline MSSA & $23(18 \%)$ & $1(5 \%)$ & ----- & $10(44 \%)$ & $12(51 \%)$ \\
\hline MRCONS & $46(37 \%)$ & $5(11 \%)$ & $5(11 \%)$ & $23(50 \%)$ & $13(28 \%)$ \\
\hline MSCONS & $5(4 \%)$ & ----- & ------ & $2(40 \%)$ & $3(60 \%)$ \\
\hline TOTAL & 125 & $14(11 \%)$ & $11(9 \%)$ & $57(46 \%)$ & $43(34 \%)$ \\
\hline
\end{tabular}

Fig.1

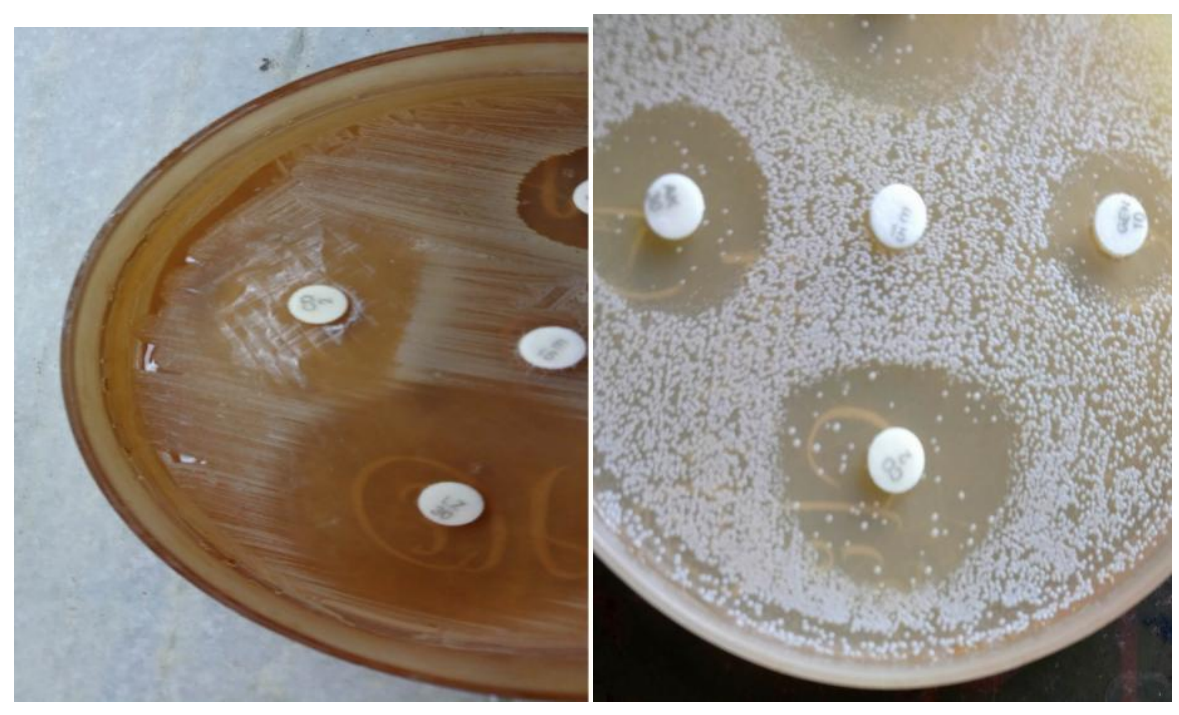

The highlight among staphylococcal infections is their emerging multidrug resistant patterns. Most of them are Methicillin Resistant Staphylococcus aureus isolates. Treatment of these infections is a difficult task for the clinicians, unless detected appropriately. Clindamycin can be the drug of choice in these situations. Cross resistance among the MLSb group is another challenging factor. A precise antibiotic susceptibility testing is of utmost importance in treating patients successfully with clindamycin. The D-test is a convenient test to detect inducible clindamycin resistance along with our routine antibiotic susceptibility testing and helps to overcome 
misinterpretation and treatment failures. It serves as a useful tool for MLSb phenotyping also. The prevalence of inducible clindamycin (iMLSb) resistance varies from area to area, and also from hospital to hospital. In the present study $11 \%$ (of the total isolates) were iMLSb isolates. Inducible resistance pattern in this study is in concordance with that reported by Sureerat Chelae et al., ${ }^{13}$ with $9.9 \%$.and Fiebelkorn et al., ${ }^{14}$ had $10 \%$ of iMLSB phenotype and Ciraj AM et al., ${ }^{15}$ from Karnataka report $13.1 \%$ of iMLSB. A north Indian study showed higher incidence of inducible resistance $(69 \%)$ compared to the present study. ${ }^{16}$ The low constitutive clindamycin resistance in this study was is in range with the study of Deotale et $a l .,{ }^{17}$ It may be due to the minimal usage of clindamycin in our hospital.

In conclusion, this study will help in documentation and understanding of inducible clindamycin resistance in a tertiary care center. This is not only important in the clinicians point of view but also for epidemiological purposes. Hence this simple, easy to perform D-test is recommended routinely for the detection of iMLSb isolates. Blind prescription of clindamycin without antibiotic susceptibility testing can lead on to treatment failures. Therefore in any hospital set up, regular surveillance of resistance pattern will be useful in formulating institutional antibiotic policies and for a better antibiotic approach.

\section{Abbreviation}

MRSA: Methicillin Resistant Staphylococcus aureus; MSSA: Methicillin Sensitive Staphylococcus aureus; MRCONS: Methicillin Resistant COagulase Negative Staphylococci; MSCONS: Methicillin Sensitive Coagulase Negative Staphylococci; iMLSB: Inducible phenotype; cMLSb: Constitutive phenotype; MSphenotype:
Erythromycin resistant Clindamycin sensitive; $S$ phenotype: Erythromycin and Clindamycin sensitive

\section{References}

1. Schito, GC., The importance of the development of antibiotic resistance in Staphylococcus aureus. Clin Microbiol Infect 2006; 12 Suppl 1: 3-8.

2. Rajaduraipandi, K., Mani KR, Panneerselvam K, Mani M, Bhaskar M, Manikandan P. Prevalence and antimicrobial susceptibility pattern of methicillin resistant Staphylococcus aureus: a multicentre study. Indian J Med Microbiol 2006; 24: 34-8].

3. Kasten, MJ., Clindamycin, metronidazole, and chloramphenicol. Mayo Clin Proc 1999; 74: 825-33.

4. Basic and clinical pharmacology $11^{\text {th }}$ edition Katzang; chapter44;799-801

5. Leclercq, R., Mechanisms of resistance to macrolides and lincosamides: Nature of the resistance elements and their clinical implications. Clin Infect Dis 2002; 34: 482-92.

6. Roberts, MC., Sutcliffe J, Courvalin P, Jensen LB, Rood J, Seppala H. Nomenclature for macrolide and macrolide-lincosamide-streptogramin B resistance determinants

7. Weisblum, B., Erythromycin resistance by ribosome modification. Antimicrob Agents Chemother 1995; 39: 577-85.

8. Clinical and Laboratory Standards Institute (CLSI). Performance Standards for Antimicrobial disk susceptibility tests: Approved Standard M2-A7. 11th ed. Clinical and Laboratory Standards Institute Wayne, $\mathrm{Pa}$; 2005.]

9. Gurdal Yilmaz, Kemalettin Aydin, Serap Iskender, Rahmet Caylan and Iftihar Koksa Detection and prevalence of inducible clindamycin resistance in staphylococci, J Med Microbiol 56 

(2007),
342-345;
DOI:

10.1099/jmm.0.46761-0 @ 2007 Society for General Microbiology.

10. Murray's Manual of clinical microbiology, $7^{\text {th }}$ edition; chapter 22.)

11. National committee for clinical laboratory standards for antimicrobial susceptibility testing sixth informational supplement 2006.M 100-S16 (M7-A7).

12. Ajantha, G.S., Ragevendra D, Kulkarni, Shetty, C. Shubhada, Pavithra Jain. Phenotypic detection of inducible clindamycin resistance among Staphylococcus aureus isolates by using limit of recommended inter -disc distance. Indian Journal of Pathology and Microbiology -51(3), JulySeptember 2008.

13. Sureerat Celae, Varaporn, Manthana, Urairat, Sineenart. Detection of inducible clindamycin resistance in staphylococci by disc diffusion induction test. J Med Assoc. Thai Vol.
92 No 2009.

14. Fiebelkorn, KR., Crawford SA, McElmeel ML, Jorgensen JH. Practical disk diffusion method for detection of inducible clindamycin resistance in Staphylococcus aureus and coagulasenegative staphylococci. J Clin Microbiol 2003; 41: 4740-4.

15. Ciraj, AM., Vinod P, Sreejith G, Rajani $\mathrm{K}$. Inducible clindamycin resistance among clinical isolates of staphylococci. Indian J Pathol Microbiol [serial online] 2009.

16. Gupta, V., Datta P, Rani H, Chander J (2009) Inducible clindamycin resistance in Staphylococcus aureus: a study from North India. J Postgrad Med 55:176179.

17. Deotale, V., Mendiratta DK, Raut U and Narang P. Inducible clindamycin resistance in Staphylococcus aureus isolated from clinical samples. Indian $\mathrm{J}$ Med Microbiol 2010; 28(2): 124-126.

\section{How to cite this article:}

Kanaga Priya Muthiah and Susitha, T. 2019. D-Test - A Useful Tool in Detection of Clindamycin Resistance among Staphylococcal Isolates in a Tertiary Care Hospital. Int.J.Curr.Microbiol.App.Sci. 8(06): 856-861. doi: https://doi.org/10.20546/ijcmas.2019.806.103 